\title{
Relationship between Decreased Mineral Intake Due to Oral Frailty and Bone Mineral Density: Findings from Shika Study
}

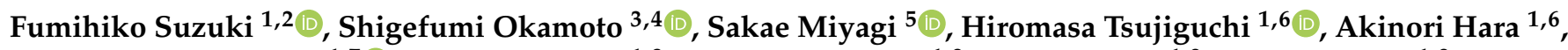 \\ Thao Thi Thu Nguyen 1,7 ${ }^{10}$, Yukari Shimizu ${ }^{1,8}$, Koichiro Hayashi ${ }^{1,9}$, Keita Suzuki ${ }^{1,9}$, Shingo Nakai ${ }^{1,9}$,

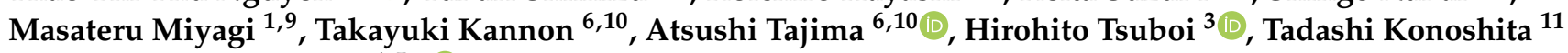 \\ and Hiroyuki Nakamura $1,5, *$ (D)
}

check for updates

Citation: Suzuki, F.; Okamoto, S.; Miyagi, S.; Tsujiguchi, H.; Hara, A.; Nguyen, T.T.T.; Shimizu, Y.; Hayashi, K.; Suzuki, K.; Nakai, S.; et al. Relationship between Decreased Mineral Intake Due to Oral Frailty and Bone Mineral Density: Findings from Shika Study. Nutrients 2021, 13, 1193. https://doi.org/10.3390/ nu13041193

Academic Editor: Sara C. Folta

Received: 26 February 2021

Accepted: 1 April 2021

Published: 5 April 2021

Publisher's Note: MDPI stays neutral with regard to jurisdictional claims in published maps and institutional affiliations.

Copyright: (c) 2021 by the authors. Licensee MDPI, Basel, Switzerland. This article is an open access article distributed under the terms and conditions of the Creative Commons Attribution (CC BY) license (https:// creativecommons.org/licenses/by/ $4.0 /)$.
1 Department of Environmental and Preventive Medicine, Graduate School of Medical Science, Kanazawa University, Kanazawa, Ishikawa 920-8640, Japan; f-suzuki@stu.kanazawa-u.ac.jp (F.S.); t-hiromasa@med.kanazawa-u.ac.jp (H.T.); ahara@m-kanazawa.jp (A.H.); toi_fs@yahoo.com (T.T.T.N.); yukari.shimizu@komatsu-u.ac.jp (Y.S.); orihciok1003@gmail.com (K.H.); keitasuzuk@stu.kanazawa-u.ac.jp (K.S.); tenhouwot@gmail.com (S.N.); okitanpopo@yahoo.co.jp (M.M.)

2 Community Medicine Support Dentistry, Ohu University Hospital, Koriyama, Fukushima 963-8611, Japan

3 Institute of Medical, Pharmaceutical and Health Sciences, Kanazawa University, Kanazawa, Ishikawa 920-1192, Japan; sokamoto@mhs.mp.kanazawa-u.ac.jp (S.O.); tsuboih@p.kanazawa-u.ac.jp (H.T.)

4 Department of Clinical Laboratory Science, Faculty of Health Sciences, Institute of Medical, Pharmaceutical and Health Sciences, Kanazawa University, Kanazawa, Ishikawa 920-0942, Japan

5 Innovative Clinical Research Center, Kanazawa University, Kanazawa, Ishikawa 920-8641, Japan; smiyagi@staff.kanazawa-u.ac.jp

6 Advanced Preventive Medical Sciences Research Center, Kanazawa University, Kanazawa, Ishikawa 920-8640, Japan; kannon@med.kanazawa-u.ac.jp (T.K.); atajima@med.kanazawa-u.ac.jp (A.T.)

7 Faculty of Public Health, Haiphong University of Medicine and Pharmacy, Ngo Quyen, Hai Phong 180000, Vietnam

8 Department of Nursing, Faculty of Health Sciences, Komatsu University, Komatsu, Ishikawa 923-0961, Japan

9 Department of Public Health, Graduate School of Advanced Preventive Medical Sciences, Kanazawa University, Kanazawa, Ishikawa 920-8640, Japan

10 Department of Bioinformatics and Genomics, Graduate School of Advanced Preventive Medical Sciences, Kanazawa University, Kanazawa, Ishikawa 920-8640, Japan

11 Third Department of Internal Medicine, Fukui University School of Medicine, Yoshida-gun, Fukui 914-0055, Japan; konosita@u-fukui.ac.jp

* Correspondence: hiro-n@po.incl.ne.jp

Abstract: The relationship between oral frailty (OF) and bone mineral density is unclear. This cross-sectional study analyzed the relationship between mineral intake and bone mineral density in middle-aged and older people with pre-oral and OF. The participants, which included 240 people aged 40 years and older, completed the three oral questions on the Kihon Checklist (KCL), which is a self-reported comprehensive health checklist, the brief-type self-administered diet history questionnaire (BDHQ), and the osteo-sono assessment index (OSI). A two-way analysis of covariance on oral function and OSI indicated that the intake of potassium, magnesium, phosphorus, squid/octopus/shrimp/shellfish, carrots/pumpkins, and mushroom was significantly lower in the $\mathrm{OF}$ and low-OSI groups than in the non-OF and high-OSI groups. A multiple logistic regression analysis for OF showed that potassium, magnesium, phosphorous and carrots/pumpkins were significantly associated with OF in the low-OSI group but not in the high-OSI group. These results demonstrated that the decrease in mineral intake due to OF was associated with decreased bone mineral density, suggesting that the maintenance of oral function prevents a decrease in bone mineral density.

Keywords: oral frailty; bone mineral density; mineral intake; osteo-sono assessment index 


\section{Introduction}

Oral frailty (OF) has been defined as the accumulation of a slightly poor status in oral conditions and function that is considered a strong prediction of physical frailty [1]. Tanaka et al. [1] reported that OF was significantly associated with 2.4-, 2.2-, 2.3-, and 2.2fold increased risk of physical frailty, sarcopenia, disability, and mortality, respectively. A previous study [2] demonstrated that the risk of frailty was associated with lower occlusal force, masseter muscle thickness, and oral diadochokinetic rate. There have been several reports on the association between systemic frailty and mineral intake [3-5]. A review by Morante et al. [3] reported that dietary factors associated with frailty were calorie, protein, vitamin $\mathrm{D}$, and calcium intake. Moreover, a cohort study [4] demonstrated that low sodium intake $(<2504 \mathrm{mg}$ ) was associated with frailty in the elderly. Furthermore, a cross-sectional study among middle- and older-aged groups suggested that dietary magnesium intake was also associated with the risk of frailty. However, few studies have evaluated the relationship between OF and mineral intake. A cross-sectional study that investigated the relationship between oral function and nutrient intake found that there was no relationship between oral health behavior (including twice a day tooth brushing or regular attendance of dental clinic) and macro- or micromineral intake [6]. However, the study did not examine systemic factors such as osteoporosis. Due to the importance of systemic factors in bone mineral density, it is necessary to evaluate its influence in the relationship between $\mathrm{OF}$ and mineral intake.

With regard to the relationship between bone mineral density and oral cavity, it has been reported that there is a relationship between decreased bone mineral density and the progression of periodontal disease, especially alveolar bone resorption in postmenopausal women [7-9]. Grocholewicza and Bohatyrewicz [7] reported a negative correlation between the lumbar bone mineral density and the periodontal disease index and between the radius bone mineral density and the papillary bleeding index. Inagaki et al. [9] also demonstrated that mineral bone density loss of metacarpal was associated with periodontitis and tooth loss in Japanese women. Therefore, osteoporosis has been considered to be a risk factor for periodontal disease. A systematic review by Gerritsen et al. [10] has shown that tooth loss is associated with impairment of oral health-related quality of life. Although it has been reported that systemic frailty is associated with reduced bone mineral density [11], it remains unclear whether $\mathrm{OF}$ is directly associated with these reductions.

On the basis of the association between OF, mineral intake, and bone mineral density, it is necessary to examine the interactions between these factors. Therefore, this study aimed to investigate the relationship between mineral intake and bone mineral density in middle-aged and older people with oral dysfunction, including pre-OF.

\section{Materials and Methods}

\subsection{Study Design and Site}

This was a cross-sectional study conducted among residents of Shika Town, Ishikawa Prefecture, Japan, termed the Shika study. As of November 2017, there were 21,007 residents in Shika Town, and 13,713 were older than 40 years [12]. The Shika study epidemiologically investigated the causes of lifestyle-related diseases through interviews, self-administered questionnaires, and comprehensive medical examinations [13-15].

\subsection{Participants}

A total of 253 people aged 40 years and older who live in four model districts (Horimatsu, Tsuchida, Higashi Matsudo, and Togi) of Shika Town provided their consent to participate in this study. Of these individuals, 13 were excluded because they did not evaluate osteo-sono assessment index (OSI) or did not have energy records within 600-4000 Kcal on the brief-type self-administered diet history questionnaire (BDHQ)). Figure 1 shows the inclusion criteria. A total of 240 participants who answered all relevant questions in the questionnaires and did not withdraw their consent were included in the analysis. 


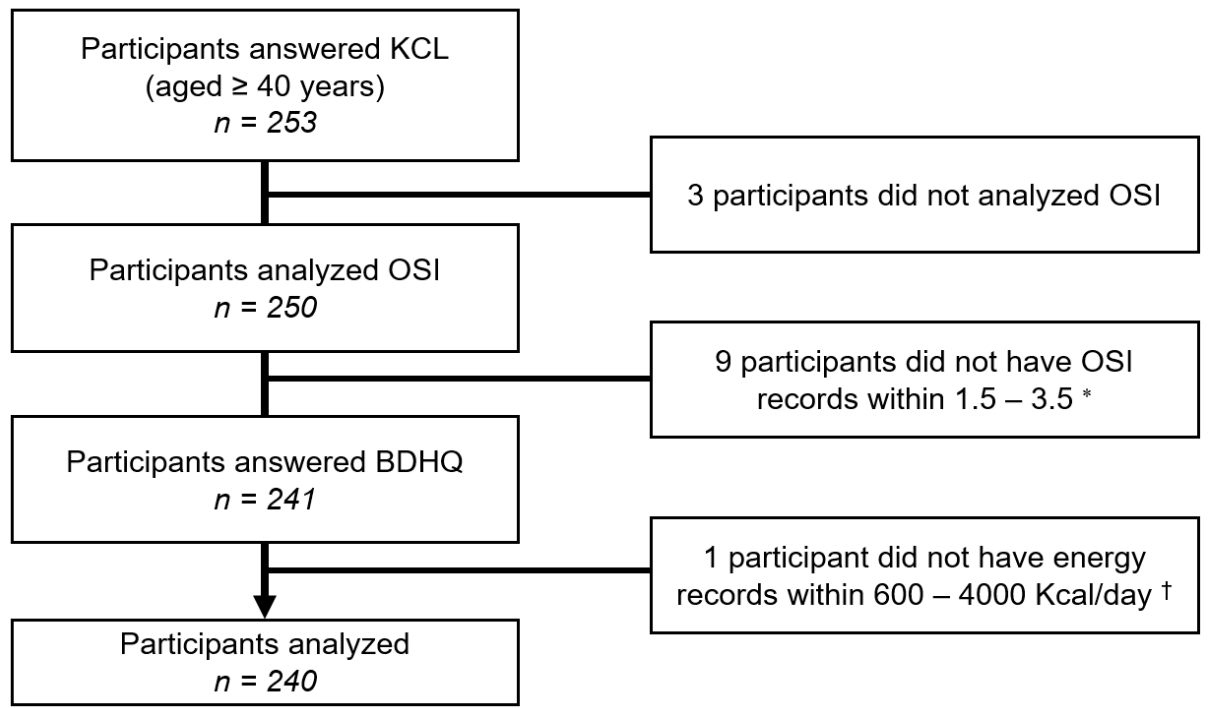

Figure 1. Participant recruitment chart. * This value was set to the OSI mean \pm 2 SD. + This reference value was chosen for the following reasons: less than $600 \mathrm{kcal} /$ day is equivalent to half the energy intake required for the lowest physical activity category, and more than $4000 \mathrm{kcal} /$ day is equivalent to 1.5 times the energy intake required for the medium physical activity category. Abbreviations: KCL, the Kihon checklist, OSI, the osteo-sono assessment index, BDHQ, the brief-type self-administered diet history questionnaire.

\subsection{Questionnaire and Measurements}

Comprehensive health survey data were collected from the residents of Shika Town, Ishikawa Prefecture, Japan, between November 2017 and February 2018. The participants completed a self-administered questionnaire on lifestyle and underlying diseases. Lifestyle items included current smokers (1. no, 2. yes) and/or current drinkers (1. no, 2. yes) and education (1. junior high school, 2. high school 3. junior college, 4 . university or higher). Underlying disease items included hypertension (1. no, 2. yes), diabetes (1. no, 2. yes), and osteoporosis (1. no, 2. yes). Body mass index (BMI) was measured using health survey data from the Shika study.

The Kihon Checklist (KCL) was used to evaluate OF. KCL is a self-reported comprehensive health checklist designed by a study group from the Ministry of Health, Labour, and Welfare as a screening tool to identify community-dwelling older adults who are vulnerable to frailty and have a higher risk of becoming dependent $[16,17]$. The validity of the KCL has been demonstrated in previous studies $[18,19]$. The three oral questions in the KCL were used as components of OF in this study. Specifically, difficulties eating tough foods from half a year ago, difficulties in swallowing tea or soup, and experience of having a dry mouth were defined as the eating, the swallowing, and the oral dryness domain, respectively. For each question, "yes" was converted to 1 point, and the total point was defined as the OF score. Furthermore, as an evaluation of oral function, we asked whether they brushed their teeth at least twice a day and recorded the current number of teeth.

Nutrient intake was assessed using the BDHQ [20,21]. The BDHQ is a four-page structured questionnaire that assesses the consumption frequency of 58 foods and beverages commonly consumed by the general Japanese population. The BDHQ estimates dietary intake in the last month using an ad hoc computer algorithm. The validity of the BDHQ has been demonstrated in previous studies $[20,21]$. To analyze nutrient data, the density method was used to estimate intake per $1000 \mathrm{Kcal}$. 
Using a quantitative ultrasonic device (AOS-100NW-B, Hitachi Aloka Medical, Tokyo, Japan), the OSI of the right calcaneus was measured as an indicator of bone strength. OSI correlates closely with bone mineral density measured by dual-energy X-ray absorptiometry [22]. OSI was calculated using the following formula:

$$
\text { OSI }=\text { transmission index } \times \text { speed of sound } 2 .
$$

\subsection{Statistical Analysis}

The participants were classified into two OF groups: the non-OF group, which had a score of 0 , and the OF group, which had a score of 1 or higher. The two OSI groups were classified into the low-OSI and high-OSI group based on the median of the participants in this study. IBM SPSS Statistics version 25 for Windows (IBM, Armonk, NY, USA) was used for the statistical analysis. Student's t-test was used to determine the association between continuous variables, while the Chi-square test was performed to investigate the association between categorical variables. A two-way analysis of covariance (ANCOVA) was used to examine the effects of the interaction between OF and OSI on mineral and food intake. The following confounding factors were adjusted for age, sex, BMI, current smoker, and current drinker. A multiple logistic regression analysis was conducted to examine the effects of OF and mineral and food intake on bone density, using the OSI as the dependent variable. In addition, the analyses were stratified by OF. Pearson's correlation coefficient was used to confirm multicollinearity. Specifically, there was no value of $|r|>0.9$ in the correlation matrix table between independent variables. The forced input method was used for variable selection. The significance level was set at $5 \%$.

\subsection{Ethics Statement}

This study was conducted with the approval of the Ethics Committee of Kanazawa University (No. 1491). Written informed consent was obtained from all participants prior to participation.

\section{Results}

\subsection{Participant Characteristics}

The participants' characteristics, OF, OSI, and mineral intakes are shown in Table 1. Of the 240 participants, 125 were males and 115 were females. Participant age ranged from 41 to 86 years, with a mean age of $60.2 \pm 10.0$ years. There was no significant difference between genders. BMI $(p<0.001)$ and OSI $(p<0.001)$ were significantly higher in males than in females. Significantly more males were current smokers, current drinkers, and better educated and had diabetes than females. On the other hand, osteoporosis was significantly higher among the females. The OF total scores were $0.7(S D=0.8)$ in both the male and female groups, with no significant difference between genders. A significantly higher number of females brushed more than twice a day than males. When comparing minerals and foods, the total energy and salt intake were significantly higher in males. Conversely, the intake of minerals, sodium, potassium, calcium, magnesium, phosphorus, green leafy vegetables, carrots/pumpkins, mushroom, and citrus was significantly higher in females. 
Table 1. Participant characteristics.

\begin{tabular}{|c|c|c|c|c|c|c|c|}
\hline & \multicolumn{2}{|c|}{ Total $(N=240)$} & \multicolumn{2}{|c|}{ Male $(n=125)$} & \multicolumn{2}{|c|}{ Female $(n=115)$} & \multirow{2}{*}{$p$-Value * } \\
\hline & Mean $(n)$ & $S D(\%)$ & Mean $(n)$ & $S D(\%)$ & Mean $(n)$ & $S D(\%)$ & \\
\hline Age, years & 60.2 & 10.0 & 59.6 & 9.2 & 60.8 & 10.8 & 0.677 \\
\hline BMI, $\mathrm{kg} / \mathrm{m}^{2}$ & 23.2 & 3.1 & 23.9 & 3.2 & 22.5 & 2.9 & $<0.001$ \\
\hline Current smoker, $n(\%)$ & 124 & 51.7 & 93 & 74.4 & 31 & 27.0 & $<0.001$ \\
\hline Current drinker, $n(\%)$ & 48 & 20.0 & 41 & 32.8 & 7 & 6.1 & $<0.001$ \\
\hline Education & & & & & & & 0.020 \\
\hline Junior high school, $n(\%)$ & 34 & 14.2 & 15 & 12.0 & 19 & 16.5 & \\
\hline High school, $n(\%)$ & 67 & 27.9 & 36 & 28.8 & 31 & 27.0 & \\
\hline Junior college, $n(\%)$ & 37 & 15.4 & 14 & 11.2 & 23 & 20.0 & \\
\hline University or higher, $n(\%)$ & 28 & 11.7 & 21 & 16.8 & 7 & 6.1 & \\
\hline Hypertension, $n(\%)$ & 78 & 32.5 & 46 & 36.8 & 32 & 27.8 & 0.162 \\
\hline Diabetes, $n(\%)$ & 17 & 7.1 & 13 & 10.4 & 4 & 3.5 & 0.045 \\
\hline Osteoporosis, $n(\%)$ & 9 & 3.8 & 1 & 0.8 & 8 & 7.0 & 0.015 \\
\hline Eating domain, $n(\%)$ & 68 & 28.3 & 41 & 32.8 & 27 & 23.5 & 0.117 \\
\hline Swallowing domain, $n(\%)$ & 53 & 22.1 & 25 & 20.0 & 28 & 24.3 & 0.440 \\
\hline Oral dryness domain, $n(\%)$ & 44 & 18.3 & 22 & 17.6 & 22 & 19.1 & 0.868 \\
\hline Oral frailty (OF), total score & 0.7 & 0.8 & 0.7 & 0.8 & 0.7 & 0.8 & 0.604 \\
\hline Number of teeth, $n(\%)$ & 18.9 & 9.4 & 18.8 & 9.0 & 19.0 & 9.8 & 0.886 \\
\hline More than twice a day brushing, $n(\%)$ & 50 & 20.8 & 15 & 12.0 & 35 & 30.4 & 0.001 \\
\hline OSI & 2.7 & 0.3 & 2.8 & 0.3 & 2.5 & 0.3 & $<0.001$ \\
\hline Total energy, Kcal & 1927.53 & 606.45 & 2128.63 & 627.81 & 1708.95 & 499.80 & $<0.001$ \\
\hline Minerals, $\%$ energy & 10.21 & 2.00 & 9.57 & 1.84 & 10.90 & 1.95 & $<0.001$ \\
\hline Sodium, $\mathrm{mg} / 1000 \mathrm{Kcal}$ & 2386.24 & 501.75 & 2336.33 & 500.57 & 2440.49 & 499.55 & $<0.001$ \\
\hline Potassium, mg/1000 Kcal & 1383.74 & 425.68 & 1195.40 & 312.30 & 1588.46 & 438.90 & $<0.001$ \\
\hline Calcium, mg/1000 Kcal & 287.38 & 106.20 & 251.89 & 95.40 & 325.96 & 104.21 & $<0.001$ \\
\hline Magnesium, mg/1000 Kcal & 138.82 & 31.52 & 126.80 & 25.62 & 151.87 & 32.22 & $<0.001$ \\
\hline Phosphorus, mg/1000 Kcal & 566.36 & 120.94 & 523.63 & 109.99 & 612.80 & 115.46 & $<0.001$ \\
\hline $\begin{array}{l}\text { Squid/octopus/shrimp/shellfish, } \\
\text { g/1000 Kcal }\end{array}$ & 16.56 & 15.73 & 17.85 & 17.02 & 15.16 & 14.13 & 0.186 \\
\hline Green leafy vegetables, g/1000 Kcal & 40.08 & 40.00 & 33.26 & 35.48 & 47.49 & 43.34 & 0.006 \\
\hline Carrots/pumpkins, g/1000 Kcal & 22.06 & 16.23 & 19.17 & 14.94 & 25.19 & 17.05 & 0.004 \\
\hline Mushroom, g/1000 Kcal & 11.75 & 9.50 & 10.01 & 8.61 & 13.65 & 10.08 & 0.003 \\
\hline Citrus, g/1000 Kcal & 21.49 & 28.45 & 16.52 & 21.85 & 26.89 & 33.49 & 0.005 \\
\hline Salt, g/1000 Kcal & 3.41 & 1.19 & 3.58 & 1.25 & 3.22 & 1.10 & 0.020 \\
\hline
\end{tabular}

* $p$-values were calculated using Student's $t$-tests and Chi-square test for continuous and categorical variables, respectively ( $p$-values less than 0.05 are highlighted in bold). Abbreviations: SD, standard deviation; BMI, body mass index; OSI, osteo-sono assessment index.

\subsection{Comparison of $O F$}

The mean age of the 115 participants in the non-OF group was 57.6 years, which was significantly lower than that of the 125 participants in the OF group (62.5 years) (Table 2). The proportion of participants with eating, swallowing, and oral dryness domain, as well as OF total score and number of teeth, were significantly higher in the OF group. When comparing each nutrient, there was no significant difference between the two groups. 
Table 2. Differences in characteristics and daily nutrient intake between the OF groups.

\begin{tabular}{|c|c|c|c|c|c|}
\hline & \multicolumn{4}{|c|}{ Total $(N=240)$} & \multirow{3}{*}{$p$-Value * } \\
\hline & \multicolumn{2}{|c|}{ Non-OF $(n=115)$} & \multicolumn{2}{|c|}{ OF $(n=125)$} & \\
\hline & Mean $(n)$ & $S D(\%)$ & Mean (n) & $S D(\%)$ & \\
\hline Age, years & 57.6 & 9.2 & 62.5 & 10.2 & $<0.001$ \\
\hline Sex (male), $n(\%)$ & 59 & 51.3 & 66 & 52.8 & 0.897 \\
\hline BMI, $\mathrm{kg} / \mathrm{m}^{2}$ & 23.4 & 3.2 & 23.1 & 3.1 & 0.354 \\
\hline Current smoker, $n(\%)$ & 58 & 50.4 & 66 & 52.8 & 0.796 \\
\hline Current drinker, $n(\%)$ & 25 & 21.7 & 23 & 18.4 & 0.524 \\
\hline Hypertension, $n(\%)$ & 32 & 27.8 & 46 & 36.8 & 0.159 \\
\hline Diabetes, $n(\%)$ & 11 & 9.6 & 6 & 4.8 & 0.207 \\
\hline Osteoporosis, $n(\%)$ & 2 & 1.7 & 7 & 5.6 & 0.174 \\
\hline Eating domain, $n(\%)$ & 0 & 0.0 & 68 & 54.4 & $<0.001$ \\
\hline Swallowing domain, $n(\%)$ & 0 & 0.0 & 53 & 42.4 & $<0.001$ \\
\hline Oral dryness domain, $n(\%)$ & 0 & 0.0 & 44 & 35.2 & $<0.001$ \\
\hline OF, total score & 0.0 & 0.0 & 1.3 & 0.5 & $<0.001$ \\
\hline Number of teeth, $n(\%)$ & 21.1 & 8.7 & 16.8 & 9.6 & $<0.001$ \\
\hline More than twice a day brushing, $n(\%)$ & 24.0 & 20.9 & 26.0 & 20.8 & 1.000 \\
\hline OSI & 2.7 & 0.3 & 2.7 & 0.3 & 0.566 \\
\hline Total energy, Kcal & 1945.59 & 628.51 & 1910.92 & 587.47 & 0.659 \\
\hline Minerals, $\%$ energy & 10.29 & 2.17 & 10.13 & 1.84 & 0.531 \\
\hline Sodium, mg/1000 Kcal & 2394.40 & 542.01 & 2378.73 & 463.73 & 0.810 \\
\hline Potassium, mg/1000 Kcal & 1412.76 & 436.21 & 1357.04 & 415.71 & 0.312 \\
\hline Calcium, mg/1000 Kcal & 287.49 & 103.61 & 287.28 & 108.94 & 0.988 \\
\hline Magnesium, mg/1000 Kcal & 140.10 & 32.61 & 137.63 & 30.56 & 0.545 \\
\hline Phosphorus, mg/1000 Kcal & 571.74 & 122.41 & 561.41 & 119.86 & 0.510 \\
\hline Squid/octopus/shrimp/shellfish, g/1000 Kcal & 16.74 & 15.98 & 16.39 & 15.55 & 0.864 \\
\hline Green leafy vegetables, g/1000 Kcal & 42.98 & 44.00 & 37.41 & 35.90 & 0.282 \\
\hline Carrots/pumpkins, g/1000 Kcal & 21.68 & 14.48 & 22.41 & 17.74 & 0.728 \\
\hline Mushroom, g/1000 Kcal & 11.75 & 9.76 & 11.75 & 9.29 & 1.000 \\
\hline Citrus, g/1000 Kcal & 24.23 & 33.21 & 18.96 & 23.09 & 0.158 \\
\hline Salt, g/1000 Kcal & 3.49 & 1.23 & 3.33 & 1.16 & 0.301 \\
\hline
\end{tabular}

${ }^{*} p$-values were calculated using Student's $t$-tests and Chi-square test for continuous and categorical variables, respectively ( $p$-values less than 0.05 are highlighted in bold). Abbreviation: $\mathrm{OF}$, oral frailty.

\subsection{Comparisons with OSI}

The mean age of the 106 participants in the low-OSI group (63.0 years) was significantly higher than that of the 134 participants in the high-OSI group (58.0 years, $p<0.001$ ) (Table 3 ). The mean OSI was significantly higher in the high-OSI group. BMI was significantly higher in the high-OSI group than in the low-OSI group. The high-OSI group significantly had a higher number of males, current smokers, current drinkers, and diabetics than the lowOSI group, whereas the low-OSI group had a significantly higher number of people with osteoporosis. When comparing each nutrient, the intake of minerals, sodium, potassium, calcium, magnesium, phosphorus, and citrus was significantly higher in the low-OSI group than in the high-OSI group, whereas that of total energy was significantly higher in the high-OSI group. 
Table 3. Differences in characteristics and daily nutrient intake between the OSI groups.

\begin{tabular}{|c|c|c|c|c|c|}
\hline & \multicolumn{4}{|c|}{ Total $(N=240)$} & \multirow{3}{*}{$p$-Value * } \\
\hline & \multicolumn{2}{|c|}{ Low OSI $(n=106)$} & \multicolumn{2}{|c|}{ High OSI $(n=134)$} & \\
\hline & Mean $(n)$ & $S D(\%)$ & Mean $(n)$ & $S D(\%)$ & \\
\hline Age, years & 63.0 & 10.5 & 58.0 & 9.1 & $<0.001$ \\
\hline Sex (male), $n(\%)$ & 29 & 27.4 & 96 & 71.6 & $<0.001$ \\
\hline BMI, $\mathrm{kg} / \mathrm{m}^{2}$ & 22.4 & 2.7 & 23.9 & 3.3 & $<0.001$ \\
\hline Current smoker, $n(\%)$ & 35 & 33.0 & 89 & 66.4 & $<0.001$ \\
\hline Current drinker, $n(\%)$ & 9 & 8.5 & 39 & 29.1 & $<0.001$ \\
\hline Hypertension, $n(\%)$ & 32 & 30.2 & 46 & 34.3 & 0.671 \\
\hline Diabetes, $n(\%)$ & 2 & 1.9 & 15 & 11.2 & 0.009 \\
\hline Osteoporosis, $n(\%)$ & 8 & 7.5 & 1 & 0.7 & 0.011 \\
\hline Eating domain, $n(\%)$ & 27 & 25.5 & 41 & 30.6 & 0.392 \\
\hline Swallowing domain, $n(\%)$ & 29 & 27.4 & 24 & 17.9 & 0.087 \\
\hline Oral dryness domain, $n(\%)$ & 25 & 23.6 & 19 & 14.2 & 0.067 \\
\hline OF, total score & 0.8 & 0.9 & 0.6 & 0.7 & 0.183 \\
\hline Number of teeth, $n(\%)$ & 18.0 & 9.3 & 19.6 & 9.4 & 0.180 \\
\hline More than twice a day brushing, $n(\%)$ & 23.0 & 21.7 & 27.0 & 20.1 & 0.873 \\
\hline OSI & 2.4 & 0.2 & 2.9 & 0.2 & $<0.001$ \\
\hline Total energy, Kcal & 1835.21 & 583.73 & 2000.56 & 616.23 & 0.036 \\
\hline Minerals, $\%$ energy & 10.80 & 2.07 & 9.73 & 1.82 & $<0.001$ \\
\hline Sodium, mg/1000 Kcal & 2467.51 & 526.16 & 2321.95 & 473.73 & 0.025 \\
\hline Potassium, mg/1000 Kcal & 1521.23 & 445.97 & 1274.98 & 376.32 & $<0.001$ \\
\hline Calcium, mg/1000 Kcal & 319.98 & 115.82 & 261.60 & 90.33 & $<0.001$ \\
\hline Magnesium, mg/1000 Kcal & 147.99 & 33.45 & 131.56 & 27.95 & $<0.001$ \\
\hline Phosphorus, mg/1000 Kcal & 604.68 & 125.66 & 536.05 & 108.27 & $<0.001$ \\
\hline Squid/octopus/shrimp/shellfish, g/1000 Kcal & 16.92 & 17.01 & 16.27 & 14.70 & 0.750 \\
\hline Green leafy vegetables, g/1000 Kcal & 43.95 & 41.26 & 37.02 & 38.86 & 0.183 \\
\hline Carrots/pumpkins, g/1000 Kcal & 23.27 & 15.81 & 21.10 & 16.56 & 0.305 \\
\hline Mushroom, g/1000 Kcal & 12.60 & 10.32 & 11.08 & 8.77 & 0.221 \\
\hline Citrus, g/1000 Kcal & 27.65 & 35.81 & 16.62 & 19.71 & 0.005 \\
\hline Salt, g/1000 Kcal & 3.37 & 1.17 & 3.44 & 1.21 & 0.639 \\
\hline
\end{tabular}

${ }^{*} p$-values were calculated using Student's $t$-tests and Chi-square test for continuous and categorical variables, respectively ( $p$-values less than 0.05 are highlighted in bold). Abbreviation: OSI, the osteo-sono assessment index.

\subsection{Main Effects and Interaction between OF and the OSI Groups}

When the non-OF group was subdivided into two groups based on OSI, there were 50 participants in the low-OSI group and 65 in the high-OSI group. The subdivision of the OF group into two groups based on the OSI similarly resulted into 56 participants in the low-OSI group and 69 in the high-OSI group (Table 4). A two-way ANCOVA adjusting for age, sex, BMI, current smoker, and current drinker was used to examine the main effects and interactions between OF and the OSI on minerals and food intake. The main effect was observed for potassium $(p=0.013)$, magnesium $(p=0.044)$, phosphorus $(p=0.047)$, green leafy vegetables $(p=0.007)$, citrus $(p=0.029)$, and salt $(p=0.043)$ intake between the two OF groups, but not between two OSI groups. Interactions were observed for sex $(p=0.037)$, $\operatorname{BMI}(p=0.013)$, number of teeth $(p=0.003)$, potassium $(p=0.038)$, magnesium $(p=0.031)$, phosphorus $(p=0.022)$, squid/octopus/shrimp/selfish $(p=0.016)$, carrots/pumpkins $(p=0.044)$, and mushroom $(p=0.045)$. A post hoc Bonferroni analysis indicated a significantly lower intake of potassium $(p=0.002)$, magnesium $(p=0.005)$, and phosphorus $(p=0.004)$ in the OF group than in the non-OF group with low OSI (Table S1, Figure S1). Similarly, there was a significantly lower intake of squid/octopus/shrimp/selfish $(p=0.029)$ and carrots/pumpkins $(p=0.037)$ in the low- OSI group than in the high-OSI group with OF (Figure S2). Table 5 shows the correlation between the minerals (potassium, magnesium, and phosphorus) and food (squid/octopus/shrimp/selfish, green leafy vegetables, carrots/pumpkins, mushroom, citrus, and salt), which indicates the main effect or the interaction in Table 4. Significant correlations between all combinations of minerals and foods, 
except squid/octopus/shrimp/selfish and potassium, were observed. This correlation demonstrated that many of the minerals and foods interacted with OF and OSI, suggesting that the decrease in bone density is related to the decrease in mineral-containing foods that are difficult to chew caused by $\mathrm{OF}$.

Table 4. Two-way ANCOVA on OF and OSI groups.

\begin{tabular}{|c|c|c|c|c|c|c|c|}
\hline & \multicolumn{4}{|c|}{ Total $(N=240)$} & \multicolumn{3}{|c|}{$p$-Value * } \\
\hline & \multicolumn{2}{|c|}{ Non-OF $(n=115)$} & \multicolumn{2}{|c|}{ OF $(n=125)$} & \multirow{3}{*}{ OF } & \multirow{3}{*}{ OSI } & \multirow{3}{*}{ OF $\times$ OSI } \\
\hline & Low-OSI $(n=50)$ & High-OSI $(n=65)$ & Low-OSI $(n=56)$ & High-OSI $(n=69)$ & & & \\
\hline & EMM (95\% CI) & EMM (95\% CI) & EMM $(95 \%$ CI) & EMM $(95 \%$ CI) & & & \\
\hline Age, years & $59.7(57.0,62.4)$ & $56.1(53.7,58.5)$ & $65.8(63.1,68.4)$ & $59.8(57.4,62.1)$ & $<0.001$ & 0.001 & 0.348 \\
\hline $\operatorname{Sex}^{+}$ & $1.4(1.3,1.6)$ & $1.6(1.5,1.7)$ & $1.3(1.2,1.4)$ & $1.7(1.6,1.8)$ & 0.858 & $<0.001$ & 0.037 \\
\hline BMI, $\mathrm{kg} / \mathrm{m}^{2}$ & $22.2(21.4,23.1)$ & $24.4(23.6,25.2)$ & $22.9(22.0,23.8)$ & $23.1(22.4,23.9)$ & 0.475 & 0.008 & 0.013 \\
\hline Current smoker $\ddagger$ & $1.1(1.0,1.2)$ & $1.3(1.2,1.4)$ & $1.1(1.0,1.2)$ & $1.2(1.1,1.3)$ & 0.722 & 0.062 & 0.751 \\
\hline Current drinker $\ddagger$ & $1.4(1.3,1.6)$ & $1.5(1.4,1.6)$ & $1.4(1.3,1.6)$ & $1.6(1.5,1.7)$ & 0.525 & 0.047 & 0.412 \\
\hline Hypertension $\ddagger$ & $1.3(1.2,1.4)$ & $1.3(1.2,1.5)$ & $1.4(1.3,1.6)$ & $1.4(1.2,1.5)$ & 0.283 & 0.896 & 0.461 \\
\hline Diabetes $\ddagger$ & $1.0(0.9,1.1)$ & $1.2(1.1,1.2)$ & $1.0(0.9,1.1)$ & $1.1(1.0,1.1)$ & 0.173 & 0.013 & 0.339 \\
\hline Osteoporosis $\ddagger$ & $1.0(1.0,1.1)$ & $1.0(1.0,1.1)$ & $1.1(1.0,1.1)$ & $1.0(1.0,1.1)$ & 0.476 & 0.360 & 0.294 \\
\hline Number of teeth, $n$ & $18.4(16.0,20.8)$ & $21.6(19.4,23.8)$ & $19.7(17.2,22.2)$ & $16.1(14.0,18.2)$ & 0.066 & 0.885 & 0.003 \\
\hline $\begin{array}{l}\text { More than twice a } \\
\text { day brushing, } n\end{array}$ & $1.2(1.0,1.3)$ & $1.2(1.1,1.3)$ & $1.1(1.0,1.3)$ & $1.3(1.2,1.4)$ & 0.785 & 0.066 & 0.691 \\
\hline Total energy, Kcal & $\begin{array}{l}1997.15(1834.08, \\
2160.22)\end{array}$ & $\begin{array}{c}1952.80(1805.82 \\
2099.78)\end{array}$ & $\begin{array}{c}1873.01(1707.91 \\
2038.10)\end{array}$ & $\begin{array}{c}1897.53(1755.37 \\
2039.70)\end{array}$ & 0.241 & 0.910 & 0.650 \\
\hline Minerals, \% energy & $10.81(10.29,11.32)$ & $10.12(9.65,10.58)$ & $9.90(9.37,10.42)$ & $10.11(9.66,10.56)$ & 0.060 & 0.392 & 0.062 \\
\hline $\begin{array}{c}\text { Sodium, mg/1000 } \\
\text { Kcal }\end{array}$ & $\begin{array}{l}2492.42(2349.10 \\
2635.74)\end{array}$ & $\begin{array}{l}2349.93(2220.75 \\
2479.11)\end{array}$ & $\begin{array}{l}2372.32(2227.22 \\
2517.42)\end{array}$ & $\begin{array}{c}2354.80(2229.86 \\
2479.74)\end{array}$ & 0.391 & 0.298 & 0.349 \\
\hline $\begin{array}{l}\text { Potassium, mg/1000 } \\
\text { Kcal }\end{array}$ & $\begin{array}{c}1500.25(1397.99 \\
1602.50)\end{array}$ & $\begin{array}{c}1393.30(1301.13 \\
1485.47)\end{array}$ & $\begin{array}{c}1281.69(1178.16 \\
1385.22)\end{array}$ & $\begin{array}{c}1373.14(1284.00 \\
1462.29)\end{array}$ & 0.013 & 0.888 & 0.038 \\
\hline $\begin{array}{c}\text { Calcium, mg/1000 } \\
\text { Kcal }\end{array}$ & $\begin{array}{l}311.00(285.15 \\
336.85)\end{array}$ & $\begin{array}{l}287.24(263.94 \\
310.54)\end{array}$ & $\begin{array}{c}268.05(241.88 \\
294.22)\end{array}$ & $\begin{array}{l}286.11(263.57 \\
308.64)\end{array}$ & 0.070 & 0.837 & 0.083 \\
\hline $\begin{array}{l}\text { Magnesium, } \\
\text { mg/1000 Kcal }\end{array}$ & $\begin{array}{c}146.78(138.89 \\
154.66)\end{array}$ & $\begin{array}{c}138.71(131.60 \\
145.81)\end{array}$ & $\begin{array}{c}131.36(123.37 \\
139.34)\end{array}$ & $\begin{array}{c}139.20(132.33 \\
146.08)\end{array}$ & 0.044 & 0.979 & 0.031 \\
\hline $\begin{array}{l}\text { Phosphorus, } \\
\text { mg/1000 Kcal }\end{array}$ & $\begin{array}{l}604.90(574.40 \\
635.40)\end{array}$ & $\begin{array}{l}559.33(531.83 \\
586.82)\end{array}$ & $\begin{array}{l}543.59(512.70 \\
574.47)\end{array}$ & $\begin{array}{l}563.54(536.95 \\
590.14)\end{array}$ & 0.047 & 0.434 & 0.022 \\
\hline $\begin{array}{l}\text { Squid/octopus/ } \\
\text { shrimp/shellfish, } \\
\text { g/1000 Kcal }\end{array}$ & $21.14(16.70,25.57)$ & $14.29(10.29,18.29)$ & $14.18(9.68,18.67)$ & $17.32(13.45,21.19)$ & 0.345 & 0.437 & 0.016 \\
\hline $\begin{array}{c}\text { Green leafy } \\
\text { vegetables, g/1000 } \\
\text { Kcal }\end{array}$ & $42.22(31.62,52.82)$ & $50.90(41.35,60.45)$ & $28.40(17.67,39.13)$ & $37.81(28.57,47.05)$ & 0.007 & 0.112 & 0.941 \\
\hline $\begin{array}{c}\text { Carrots/pumpkins, } \\
\text { g/1000 Kcal }\end{array}$ & $23.49(18.97,28.01)$ & $21.80(17.72,25.87)$ & $17.86(13.29,22.44)$ & $24.67(20.73,28.61)$ & 0.515 & 0.292 & 0.044 \\
\hline $\begin{array}{l}\text { Mushroom, g/1000 } \\
\text { Kcal }\end{array}$ & $12.79(10.14,15.44)$ & $11.80(9.41,14.19)$ & $9.11(6.43,11.80)$ & $13.10(10.78,15.41)$ & 0.339 & 0.293 & 0.045 \\
\hline Citrus, g/1000 Kcal & $32.57(24.70,40.44)$ & $19.64(12.54,26.73)$ & $17.90(9.93,25.87)$ & $18.12(11.26,24.98)$ & 0.029 & 0.133 & 0.073 \\
\hline Salt, g/1000 Kcal & $3.74(3.40,4.07)$ & $3.42(3.13,3.72)$ & $3.15(2.82,3.49)$ & $3.38(3.09,3.67)$ & 0.043 & 0.806 & 0.083 \\
\hline
\end{tabular}


Table 5. Correlation between minerals and foods.

\begin{tabular}{|c|c|c|c|c|c|c|}
\hline & $\begin{array}{l}\text { Squid/Octopus/ } \\
\text { Shrimp/Shellfish, } \\
\text { g/1000 Kcal }\end{array}$ & $\begin{array}{l}\text { Green Leafy } \\
\text { Vegetables, } \\
\text { g/1000 Kcal }\end{array}$ & $\begin{array}{l}\text { Carrots/Pumpkins, } \\
\text { g/1000 Kcal }\end{array}$ & $\begin{array}{l}\text { Mushroom, } \\
\text { g/1000 Kcal }\end{array}$ & $\begin{array}{c}\text { Citrus, } \\
\text { g/1000 Kcal }\end{array}$ & $\begin{array}{l}\text { Salt, g/1000 } \\
\text { Kcal }\end{array}$ \\
\hline Minerals, \% energy & $0.226(<\mathbf{0 . 0 0 1 )} *$ & $0.415(<0.001)$ & $0.265(<0.001)$ & $0.325(<\mathbf{0 . 0 0 1 )}$ & 0.194 (0.003) & $0.239(<0.001)$ \\
\hline Sodium, mg/1000 Kcal & $0.226(<\mathbf{0 . 0 0 1 )}$ & $0.105(0.105)$ & $-0.112(0.085)$ & $0.070(0.279)$ & $0.041(0.527)$ & $0.192(0.003)$ \\
\hline Potassium, mg/1000 Kcal & $0.123(0.057)$ & $0.618(<0.001)$ & $0.604(<0.001)$ & $0.478(<\mathbf{0 . 0 0 1})$ & 0.305 (<0.001) & $0.184(\mathbf{0 . 0 0 4 )}$ \\
\hline Calcium, mg/1000 Kcal & $0.125(0.053)$ & $0.486(<0.001)$ & $0.400(<0.001)$ & $0.375(<\mathbf{0 . 0 0 1 )}$ & 0.225 (<0.001) & 0.177 (0.006) \\
\hline Magnesium, mg/1000 Kcal & $0.226(<0.001)$ & $0.590(<0.001)$ & $0.521(<0.001)$ & $0.472(<\mathbf{0 . 0 0 1 )}$ & $0.277(<\mathbf{0 . 0 0 1 )}$ & $0.232(<0.001)$ \\
\hline Phosphorus, mg/1000 Kcal & $0.289(<0.001)$ & $0.434(<0.001)$ & $0.416(<0.001)$ & $0.437(<\mathbf{0 . 0 0 1 )}$ & $0.235(<\mathbf{0 . 0 0 1 )}$ & $0.384(<\mathbf{0 . 0 0 1 )}$ \\
\hline
\end{tabular}

* Pearson's correlation coefficient ( $p$-value). $p$-values less than 0.05 are highlighted in bold.

\subsection{Effects of OF and Mineral and Food Intake on OSI}

Table 6 shows the results of multiple logistic regression analysis stratified by OSI with $\mathrm{OF}$ as the dependent variable and mineral intake as the independent variable. Minerals (OR: $0.767 ;$ 95\% CI: 0.621, 0.948; $p=0.014$ ), potassium (OR: 0.998; 95\% CI: 0.997, 0.999; $p=0.004$ ), calcium (OR: 0.995; 95\% CI: 0.991, 0.999; $p=0.028)$, magnesium (OR: 0.981; 95\% CI: $0.968,0.994 ; p=0.006$ ), and phosphorus (OR: $0.995 ; 95 \%$ CI: $0.991,0.998 ; p=0.004$ ) were significant independent variables in the model with adjustment for age, sex, BMI, current smoker, and current drinker in the low-OSI group but not in the high-OSI group. This result implies that bone density is negatively associated with low mineral intake only in the low-OSI group with OF. Table 7 shows the results of the same analysis and confounding factor adjustment but with food as the independent variable. Carrots/pumpkins (OR: $0.971 ; 95 \%$ CI: $0.944,1.000 ; p=0.047$ ), citrus (OR: 0.986; 95\% CI: 0.974, 0.999; $p=0.038$ ), and salt (OR: $0.601 ; 95 \%$ CI: 0.410, 0.882; $p=0.009$ ) were significant independent variables in the model in the low-OSI group but not in the high-OSI group. This result implies that bone density is negatively associated with low carrots/pumpkins, citrus, and salt intake only in the low-OSI group with OF.

Table 6. Relationship between mineral intake and OF stratified by OSI.

\begin{tabular}{ccccccc}
\hline & & & & & \multicolumn{2}{c}{95 \% CI } \\
\cline { 5 - 7 } & & $\beta$ & $p$-Value & OR & Lower & Upper \\
\hline \multirow{2}{*}{ Low-OSI group } & Minerals & -0.265 & $\mathbf{0 . 0 1 4}$ & 0.767 & 0.621 & 0.948 \\
& Sodium & -0.001 & 0.193 & 0.999 & 0.999 & 1.000 \\
& Potassium & -0.002 & $\mathbf{0 . 0 0 4}$ & 0.998 & 0.997 & 0.999 \\
& Calcium & -0.005 & $\mathbf{0 . 0 2 8}$ & 0.995 & 0.991 & 0.999 \\
& Magnesium & -0.019 & $\mathbf{0 . 0 0 6}$ & 0.981 & 0.968 & 0.994 \\
& Phosphorus & -0.005 & $\mathbf{0 . 0 0 4}$ & 0.995 & 0.991 & 0.998 \\
\hline High-OSI group & Minerals & -0.014 & 0.890 & 0.986 & 0.812 & 1.199 \\
& Sodium & 0.000 & 0.909 & 1.000 & 0.999 & 1.001 \\
& Potassium & 0.000 & 0.747 & 1.000 & 0.999 & 1.001 \\
& Calcium & 0.000 & 0.990 & 1.000 & 0.996 & 1.004 \\
& Magnesium & 0.000 & 0.991 & 1.000 & 0.987 & 1.013 \\
& Phosphorus & 0.000 & 0.960 & 1.000 & 0.997 & 1.003
\end{tabular}

Significant estimates are in bold. Adjusted for age, sex, body mass index (BMI), current smoker, and current drinker. Abbreviations: OR, odds ratio. 
Table 7. Relationship between food intake and OF stratified by OSI.

\begin{tabular}{|c|c|c|c|c|c|c|}
\hline & & \multirow{2}{*}{$\beta$} & \multirow{2}{*}{$p$-Value } & \multirow{2}{*}{ OR } & \multicolumn{2}{|c|}{$95 \% \mathrm{CI}$} \\
\hline & & & & & Lower & Upper \\
\hline \multirow[t]{6}{*}{ Low-OSI group } & Squid/octopus/shrimp/shellfish & -0.027 & 0.059 & 0.973 & 0.946 & 1.001 \\
\hline & Green leafy vegetables & -0.010 & 0.078 & 0.990 & 0.979 & 1.001 \\
\hline & Carrots/pumpkins & -0.029 & 0.047 & 0.971 & 0.944 & 1.000 \\
\hline & Mushroom & -0.042 & 0.052 & 0.959 & 0.919 & 1.000 \\
\hline & Citrus & -0.014 & 0.038 & 0.986 & 0.974 & 0.999 \\
\hline & Salt & -0.509 & 0.009 & 0.601 & 0.410 & 0.882 \\
\hline \multirow[t]{6}{*}{ High-OSI group } & Squid/octopus/shrimp/shellfish & 0.017 & 0.189 & 1.018 & 0.991 & 1.045 \\
\hline & Green leafy vegetables & -0.009 & 0.087 & 0.991 & 0.981 & 1.001 \\
\hline & Carrots/pumpkins & 0.014 & 0.232 & 1.015 & 0.991 & 1.039 \\
\hline & Mushroom & 0.015 & 0.496 & 1.015 & 0.972 & 1.059 \\
\hline & Citrus & -0.005 & 0.616 & 0.995 & 0.976 & 1.015 \\
\hline & Salt & -0.045 & 0.784 & 0.956 & 0.696 & 1.315 \\
\hline
\end{tabular}

Significant estimates are in bold. Adjusted for age, sex, BMI, current smoker, and current drinker.

\section{Discussion}

Since oral frailty is a relatively new concept, its evaluation method is not well established. In the long-term care insurance system for the elderly in Japan, three domains related to the oral cavity of the KCL are used for the evaluation items of oral dysfunction $[18,19]$. In addition to these three domains, the current number of teeth $[1,23]$, masticatory ability [1], tooth brushing twice daily [6], regular dental visits [6], denture use [24], occlusal force [2], tongue-lip function [2], and eating alone [25] were used to evaluate OF. Furthermore, since the evaluation method of pre-OF has not been fully discussed, there have been some reports $[25,26]$ that the survey items of OF with few applicable items are regarded as pre-OF. In this study, we decided to use three domains related to the oral cavity in the KCL to evaluate the oral dysfunction from an early stage, including pre-OF, as a population approach.

The main result of this study was that when OSI was stratified into two groups with $\mathrm{OF}$ as the dependent variable in multiple logistic regression analysis, minerals, potassium, calcium, magnesium, phosphorus, carrots/pumpkins, citrus, and salt intake were found to be significant independent variables in the low-OSI group.

According to ANCOVA results, the main effects were observed in potassium, magnesium, phosphorus, green leafy vegetables, citrus, and salt intake even after adjusting for confounding factors in the OF group. This contrasted the study by Nomura et al. [6], who investigated the relationship between $\mathrm{OF}$ and nutrient intake and found no association between $\mathrm{OF}$ and mineral intake. They evaluated factors related to oral health behavior and macronutrients using structural equation modeling. It seems that the reason why our results differed from theirs was that they did not analyze the involvement of chewing and swallowing functions in the assessment of food choice and oral dysfunction. Alternatively, Zhe et al. [23] reported that protein, vitamins, and mineral intake is positively associated with the total number of natural teeth. This study was thought to have elucidated the non-oral frailty and high-OSI group had 20 or more current teeth, while the other groups had less than 20 current teeth, indicating an interaction. In other words, it indicates that there is a relationship between the decline in oral function and food choice.

It has also been noted that not nutrients or foods, but $\mathrm{HbA} 1 \mathrm{c}$, diabetes, and currently drunk showed a main effect on the OSI even after adjusting the covariates. A meta-analysis by Ma et al. [27] and a review by Piepkorn et al. [28] reported that patients with type 2 diabetes have higher bone mineral density than non-diabetic patients. Our results that showed that the proportion of patients with diabetes was higher in the high-OSI group support this finding. A prospective study by Holbrook and Barrett-Connor [29] also reported that drinking increased bone mineral density. Our findings that the proportion of current drinkers was higher in the high-OSI group might also support the report.

Potassium, magnesium, phosphorus, carrots/pumpkins, citrus, and salt intake were observed to have the main effect on the two OF groups or the interaction among the four OF and OSI groups. These minerals and foods were also significant independent 
variables when stratified by OSI in multiple logistic regression analysis with OF as the dependent variable. In line with our finding, previous studies investigating the relationship between mineral intake and bone mineral density reported that potassium and magnesium intake was associated with increased bone mineral density in the elderly [30]; that dietary protein, phosphorus, and potassium were beneficial to bone mineral density in adult men consuming adequate dietary calcium [31]; and that magnesium intake was associated with bone mineral density [5,32]. With regard to the relationship between food intake and bone mineral density, Kim et al. [33] reported that intake of anchovies, radishes, carrots, zucchinis, and tomatoes were significant important factors in minimizing bone density risk. Our results support the above findings because we found a similar relationship between the minerals or mineral-containing food intake and OSI. Our study showed that the mean current number of teeth was 20 or more only in the non-OF and high-OSI group. Poor chewing and swallowing function due to OF and having less than 20 current teeth are presumed to reduce the variety of chewable foods. It suggests that the decrease in mineral intake due to the change in the food choice by OF is associated with the decrease in bone mineral density.

Since this study was a cross-sectional study, the causal relationship between OF, mineral intake, and bone mineral density could not be ascertained. Moreover, OSI does not directly evaluate bone mineral density. Finally, self-administration of BDHQ may lack objectivity.

\section{Conclusions}

Our study demonstrated a decrease in mineral intake due to OF, which was associated with decreased bone mineral density. These results suggest that the maintenance of oral function prevents decrease in bone mineral density.

Supplementary Materials: The following are available online at https://www.mdpi.com/article/ 10.3390/nu13041193/s1, Table S1: Post hoc analysis of interacting nutrients and foods. Multiple comparisons using the Bonferroni analysis (p-values less than 0.05 are highlighted in bold), Figure S1: Interaction of OF and the OSI groups on minerals intake. Estimated marginal means were adjusted based on the value age $=60.2$, sex $=1.5, \mathrm{BMI}=23.2$, current smoker $=1.20$, and current drinker $=$ 1.52, Figure S2: Interaction of OF and the OSI groups on foods intake. Estimated marginal means were adjusted based on the value age $=60.2, \operatorname{sex}=1.5, \mathrm{BMI}=23.2$, current smoker $=1.20$, and current drinker $=1.52$.

Author Contributions: Conceptualization, F.S. and H.N.; Data curation, F.S., S.O., S.M., H.T. (Hiromasa Tsujiguchi), A.H., T.T.T.N., Y.S., K.H., K.S., S.N., M.M., T.K. (Takayuki Kannon), A.T., H.T. (Hirohito Tsuboi), T.K. (Tadashi Konoshita) and H.N.; Formal analysis, F.S., S.O., S.M., H.T. (Hiromasa Tsujiguchi), A.H., T.T.T.N., Y.S., K.H., K.S., S.N., M.M., T.K. (Takayuki Kannon), A.T., H.T. (Hirohito Tsuboi), T.K. (Tadashi Konoshita) and H.N.; Funding acquisition, H.N.; Investigation, F.S., S.O., S.M., H.T. (Hiromasa Tsujiguchi), A.H., T.T.T.N., Y.S., K.H., K.S., S.N., M.M., T.K. (Takayuki Kannon), A.T., H.T. (Hirohito Tsuboi), T.K. (Tadashi Konoshita) and H.N.; Project administration, F.S. and H.N.; Resources, H.N.; Supervision, H.N.; Validation, F.S., S.O., S.M., H.T. (Hiromasa Tsujiguchi), A.H., T.T.T.N., Y.S., K.H., K.S., S.N., M.M., T.K. (Takayuki Kannon), A.T., H.T. (Hirohito Tsuboi), T.K. (Tadashi Konoshita) and H.N.; Writing — original draft, F.S. All authors have read and agreed to the published version of the manuscript.

Funding: This study was supported by a Grant-in-Aid for Scientific Research (B) by the Ministry of Education, Culture, Sport, Science and Technology (MEXT), number 15H04783.

Institutional Review Board Statement: The study was conducted in accordance with the Declaration of Helsinki and approved by the Ethics Committee of Kanazawa University (protocol code, 1491; date of approval, 18 December 2013).

Informed Consent Statement: Written informed consent to publish this paper was obtained from the participants.

Data Availability Statement: Available on request and by approval (Kanazawa University Ethics Committee. Person in charge: Yuko Katsuragi email: pub-jim2@staff.kanazawa-u.ac.jp. 
Acknowledgments: The authors thank all the participants in the Shika Town study, as well as the collaborators including the field survey staff and experimental staff.

Conflicts of Interest: Financial disclosure statement: The funders had no role in the study design, data collection and analysis, decision to publish, or preparation of the manuscript. Non-financial disclosure statement: The authors declare no conflict of interest associated with this manuscript.

\section{References}

1. Tanaka, T.; Takahashi, K.; Hirano, H.; Kikutani, T.; Watanabe, Y.; Ohara, Y.; Furuya, H.; Tetsuo, T.; Akishita, M.; Iijima, K. Oral frailtyasa risk factor for physical frailty and mortality in community-dwelling elderly. J. Gerontol. Ser. A Biol. Sci. Med. Sci. 2018, 73, 1661-1667. [CrossRef]

2. Watanabe, Y.; Hirano, H.; Arai, H.; Morishita, S.; Ohara, Y.; Edahiro, A.; Murakami, M.; Shimada, H.; Kikutani, T.; Suzuki, T. Relationship Between Frailty and Oral Function in Community-Dwelling Elderly Adults. J. Am. Geriatr. Soc. 2017, 65, 66-76. [CrossRef]

3. Hernández Morante, J.J.; Gómez Martínez, C.; Morillas-Ruiz, J.M. Dietary Factors Associated with Frailty in Old Adults: A Review of Nutritional Interventions to Prevent Frailty Development. Nutrients 2019, 11, 102. [CrossRef] [PubMed]

4. Kim, S.; Kim, M.; Min, J.; Yoo, J.; Kim, M.; Kang, J.; Won, C.W. How much intake of sodium is good for frailty? The Korean Frailty and Aging Cohort Study (KFACS). J. Nutr. Health Aging 2019, 23, 503-508. [CrossRef] [PubMed]

5. Welch, A.A.; Skinner, J.; Hickson, M. Dietary magnesium may be protective for aging of bone and skeletal muscle in middle and younger older age men and women: Cross-sectional findings from the UK biobank cohort. Nutrients 2017, 9, 1189. [CrossRef] [PubMed]

6. Nomura, Y.; Ishii, Y.; Suzuki, S.; Morita, K.; Suzuki, A.; Suzuki, S.; Tanabe, J.; Ishiwata, Y.; Yamakawa, K.; Chiba, Y.; et al. Nutritional Status and Oral Frailty: A Community Based Study. Nutrients 2020, 12, 2886. [CrossRef]

7. Grocholewicz, K.; Bohatyrewicz, A. Oral health and bone mineral density in postmenopausal women. Arch. Oral Biol. 2012, 57, 245-251. [CrossRef] [PubMed]

8. Inagaki, K.; Kurosu, Y.; Kamiya, T.; Kondo, F.; Yoshinari, N.; Noguchi, T.; Krall, E.A.; Garcia, R.I. Low metacarpal bone density, tooth loss, and periodontal disease in Japanese women. J. Dent. Res. 2001, 80, 1818-1822. [CrossRef] [PubMed]

9. Inagaki, K.; Kurosu, Y.; Sakano, M.; Yamamoto, G.; Kikuchi, T.; Noguchi, T.; Yano, H.; Izawa, H.; Hachiya, Y. Oral osteoporosis: A review and its dental implications. Clin. Calcium 2007, 17, 157-163. [PubMed]

10. Gerritsen, A.E.; Allen, P.F.; Witter, D.J.; Bronkhorst, E.M.; Creugers, N.H.J. Tooth loss and oral health-related quality of life: A systematic review and meta-analysis. Health Qual. Life Outcomes 2010, 8, 1-11. [CrossRef]

11. Villareal, D.T.; Binder, E.F.; Yarasheski, K.E.; Williams, D.B.; Schechtman, K.B.; Kohrt, W.M. Bone mineral density response to estrogen replacement in frail elderly women; A randomized controlled trial. J. Am. Med. Assoc. 2001, 286, 815-820. [CrossRef] [PubMed]

12. Shika Town Population. Available online: https://www.town.shika.lg.jp/jyuumin/shika_town_pop/heisei29/shikatown_pop_ 201711.html (accessed on 11 June 2020).

13. Tsujiguchi, H.; Nguyen, T.T.T.; Goto, D.; Miyagi, S.; Kambayashi, Y.; Hara, A.; Yamada, Y.; Nakamura, H.; Shimizu, Y.; Hori, D.; et al. Relationship between the intake of $n-3$ polyunsaturated fatty acids and depressive symptoms in elderly japanese people: Differences according to sex and weight status. Nutrients 2019, 11, 775. [CrossRef]

14. Nakamura, H.; Tsujiguchi, H.; Kambayashi, Y.; Hara, A.; Miyagi, S.; Yamada, Y.; Nguyen, T.T.T.; Shimizu, Y.; Hori, D.; Nakamura, H. Relationship between saturated fatty acid intake and hypertension and oxidative stress. Nutrition 2019, 61, 8-15. [CrossRef] [PubMed]

15. Tsujiguchi, H.; Hori, D.; Kambayashi, Y.; Hamagishi, T.; Asakura, H.; Mitoma, J.; Kitaoka, M.; Anyenda, E.O.; Nguyen, T.T.T.; Yamada, Y.; et al. Relationship between screen time and nutrient intake in Japanese children and adolescents: A cross-sectional observational study. Environ. Health Prev. Med. 2018, 23. [CrossRef] [PubMed]

16. Nemoto, M.; Yabushita, N.; Kim, M.J.; Matsuo, T.; Seino, S.; Tanaka, K. Assessment of vulnerable older adults' physical function according to the Japanese Long-Term Care Insurance (LTCI) system and Fried's criteria for frailty syndrome. Arch. Gerontol. Geriatr. 2012, 55, 385-391. [CrossRef] [PubMed]

17. Japanese Ministry of Health, Labour and Welfare. The Manuals of the Evaluation for Ability to Perform Daily Activities on Preventive Care. Available online: https:/ /www.mhlw.go.jp/topics/2009/05/dl/tp0501-1c_0001.pdf (accessed on 23 November 2020).

18. Satake, S.; Senda, K.; Hong, Y.J.; Miura, H.; Endo, H.; Sakurai, T.; Kondo, I.; Toba, K. Validity of the Kihon Checklist for assessing frailty status. Geriatr. Gerontol. Int. 2016, 16, 709-715. [CrossRef] [PubMed]

19. Satake, S.; Shimokata, H.; Senda, K.; Kondo, I.; Toba, K. Validity of Total Kihon Checklist Score for Predicting the Incidence of 3-Year Dependency and Mortality in a Community-Dwelling Older Population. J. Am. Med Dir. Assoc. 2017, 18, 552.e1-552.e6. [CrossRef] [PubMed]

20. Kobayashi, S.; Murakami, K.; Sasaki, S.; Okubo, H.; Hirota, N.; Notsu, A.; Fukui, M.; Date, C. Comparison of relative validity of food group intakes estimated by comprehensive and brief-type self-administered diet history questionnaires against $16 \mathrm{~d}$ dietary records in Japanese adults. Public Health Nutr. 2011, 14, 1200-1211. [CrossRef] [PubMed] 
21. Kobayashi, S.; Honda, S.; Murakami, K.; Sasaki, S.; Okubo, H.; Hirota, N.; Notsu, A.; Fukui, M.; Date, C. Both comprehensive and brief self-administered diet history questionnaires satisfactorily rank nutrient intakes in Japanese adults. J. Epidemiol. 2012, 22, 151-159. [CrossRef]

22. Sasaki, M.; Harata, S.; Kumazawa, Y.; Mita, R.; Kida, K.; Tsuge, M. Bone mineral density and osteo sono assessment index in adolescents. J. Orthop. Sci. 2000, 5, 185-191. [CrossRef]

23. Zhu, Y.; Hollis, J.H. Tooth loss and its association with dietary intake and diet quality in American adults. J. Dent. 2014, 42, 1428-1435. [CrossRef] [PubMed]

24. Semba, R.D.; Blaum, C.S.; Bartali, B.; Xue, Q.L. Denture use, malnutrition, frailty, and mortality among older women living in the community-PubMed. J. Nutr. Health Aging 2006, 10, 161-167. [PubMed]

25. Ohara, Y.; Motokawa, K.; Watanabe, Y.; Shirobe, M.; Inagaki, H.; Motohashi, Y.; Edahiro, A.; Hirano, H.; Kitamura, A.; Awata, S.; et al. Association of eating alone with oral frailty among community-dwelling older adults in Japan. Arch. Gerontol. Geriatr. 2020, 87. [CrossRef] [PubMed]

26. Hoshino, D.; Hirano, H.; Edahiro, A.; Motokawa, K.; Shirobe, M.; Watanabe, Y.; Motohashi, Y.; Ohara, Y.; Iwasaki, M.; Maruoka, Y.; et al. Association Between Oral Frailty and Dietary Variety Among Community-Dwelling Older Persons: A Cross-Sectional Study. J. Nutr. Health Aging 2020, 1-8. [CrossRef]

27. Ma, L.; Oei, L.; Jiang, L.; Estrada, K.; Chen, H.; Wang, Z.; Yu, Q.; Zillikens, M.C.; Gao, X.; Rivadeneira, F. Association between bone mineral density and type 2 diabetes mellitus: A meta-Analysis of observational studies. Eur. J. Epidemiol. 2012, 27, 319-332. [CrossRef]

28. Piepkorn, B.; Kann, P.; Forst, T.; Andreas, J.; Pfützner, A.; Beyer, J. Bone Mineral Density and Bone Metabolism in Diabetes Mellitus. Horm. Metab. Res. 1997, 29, 584-591. [CrossRef]

29. Holbrook, T.L.; Barrett-Connor, E. A prospective study of alcohol consumption and bone mineral density. Br. Med. J. 1993, 306, 1506-1509. [CrossRef]

30. Tucker, K.L.; Hannan, M.T.; Chen, H.; Cupples, L.A.; Wilson, P.W.; Kiel, D.P. Potassium, magnesium, and fruit and vegetable intakes are associated with greater bone mineral density in elderly men and women. Am. J. Clin. Nutr. 1999, 69, 727-736. [CrossRef]

31. Whiting, S.J.; Boyle, J.L.; Thompson, A.; Mirwald, R.L.; Faulkner, R.A. Dietary protein, phosphorus and potassium are beneficial to bone mineral density in adult men consuming adequate dietary calcium. J. Am. Coll. Nutr. 2002, 21, 402-409. [CrossRef]

32. Orchard, T.S.; Larson, J.C.; Alghothani, N.; Bout-Tabaku, S.; Cauley, J.A.; Chen, Z.; LaCroix, A.Z.; Wactawski-Wende, J.; Jackson, R.D. Magnesium intake, bone mineral density, and fractures: Results from the Women's Health Initiative Observational Study. Am. J. Clin. Nutr. 2014, 99, 926-933. [CrossRef]

33. Kim, M.S.; Koo, J.O. Analysis of factors affecting bone mineral density with different age among adult women in Seoul area. Korean J. Community Nutr. 2007, 12, 559-568. 